\title{
Sequências numéricas e cálculo de probabilidades associadas a modelos de urnas
}

Valdivino Vargas Júnior iD
Divaldo P. Fernandes Júnior (D)

\section{Resumo}

Considere uma urna contendo bolas diferindo apenas por suas cores. Dessa urna, bolas são extraídas ao acaso. No caso mais simples, a urna contém bolas de duas cores. Essas são extraídas de forma aleatória, podendo ou não haver reposição. No mais geral, podem-se estabelecer diversas regras: acrescentar bolas ao longo do processo de acordo com algum critério, trabalhar com bolas de diversas cores, fazer intercâmbio de bolas de uma urna para outra etc. Neste artigo, apresentamos algumas situações nas quais o cálculo probabilístico é feito via propriedades de sequências numéricas.

Palavras-chave: Modelos de Urnas; Sequências Numéricas; Probabilidade.

\section{Abstract}

Consider an urn containing balls differing only in their colors. From this urn, balls are extracted at random. In the simplest case, the urn contains balls of two colors. These are extracted at random and may or may not be replaced. In general, several rules can be established: adding balls along the process according to some criteria, working with balls of different colors, exchanging balls from one urn to another, etc. In this article, we present some situations in which the probabilistic calculation is done through number sequence properties.

keywords: Urns Models; Numeric Sequences; Probability.

\section{Introdução}

A maioria dos processos que vivenciamos no nosso cotidiano envolvem algum tipo de aleatoriedade. Com base nisso, torna-se fundamental que estudantes dominem conceitos básicos envolvendo experimentos aleatórios. Em termos de Ensino Básico, o ensino de probabilidade é encarado como a competência na qual deve ser desenvolvida a compreensão da natureza aleatória da maioria dos fenômenos naturais e sociais. O cálculo probabilístico provê ferramentas adequadas para mensurar as incertezas associadas aos fenômenos aleatórios. Os modelos de urna apresentam problemas cujas situações descritas auxiliam a compreeensão básica de conceitos de probabilidade. Na versão mais simples de um modelo de urnas, temos uma urna com bolas que diferem apenas por suas cores. Extrações são realizadas ao acaso. Numa versão, anota-se a cor da bola retirada e, logo a seguir é devolvida à urna antes da próxima extração. Noutra versão, as bolas extraídas não são devolvidas. O interesse, nesses casos, é a distribuição de probabilidade do número de bolas extraídas de uma 
das cores. A partir dessas versões básicas surgem modelos mais elaborados. Em alguns, há acréscimo de bolas entre as retiradas de acordo com alguma regra específica. E, em geral, trabalha-se com bolas de diversas cores.

Heubeck (1974) cita que a primeira referência ao uso de urnas em problemas de probabilidade apareceu nos trabalhos de Huygens (1629-1695). Embora ele não cite a expressão modelo de urnas, muitas de suas contas podem ser expressas no contexto de problemas de urnas. Alguns anos depois, menções a modelos de urnas aparecem no trabalho de J. Bernoulli (1654-1705), em Ars Conjectandi (livro III), publicado postumamente em 1713. Alguns anos depois, D. Bernoulli (1700-1782), por diversas vezes, recorre a modelos de urnas. Em particular, em um trabalho de 1768, D. Bernoulli propôs um modelo, hoje conhecido como modelo de Ehrenfest. Modelos de urnas aparecem ainda em outros trabalhos de importantes matemáticos da época, dos quais podemos destacar: De Moivre (1667-1754) e Montmort (1678-1719). Algum tempo mais tarde, Laplace (1749-1827) estudou em detalhes o modelo de Ehrenfest. No século XIX modelos de urnas apareceram em diversos trabalhos de probabilistas e estatísticos da época. Dentre os autores que voltaram a atenção para esse tipo de modelo podemos citar: Quetelet (1794-1874) e Poisson (1781-1840). No início do século XX, mais especificamente em 1906, Markov (1856-1922) introduziu os modelos que hoje são chamados modelos de Pólya-Eggenberger. Para maiores detalhes sobre modelagem com urnas e sua evolução histórica veja Johnson e Kotz (1977).

Dentre as modelagens com urnas, têm destaque processos onde se estudam as distribuições do número de bolas em urnas. Esses processos, chamados modelos de ocupação, são definidos em diferentes vertentes no que tange às regras de distribuição das bolas, número de urnas, tipos de urnas etc. Sua origem ainda é razão de investigação, pois remontam a vários séculos atrás. Sprott (1956) cita que esse tipo de modelo já aparecia nos trabalhos de De Moivre (1718), e em versões mais generalizadas em conexão com problemas da física teórica nos trabalhos de Clausius (18221888), Boltzmann (1844-1906) e Maxwell (1831-1879) sobre teoria dos gases. Mais detalhes sobre modelos de ocupação podem ser encontrados em Charalambides (2005).

Os modelos de urna possuem muitas aplicações práticas. Pode-se imaginar que as bolas constituem uma população de indivíduos. As características de interesse naquela população são representadas pelas cores das bolas. As extrações constituem o processo de amostragem. Assim, esses modelos ajudam na compreensão do funcionamento básico de um processo de amostragem. Em situações práticas os processos de amostragem antecedem os procedimentos de inferência estatística. Basicamente, fazer inferência estatística é, a partir de dados coletados, estimar e tomar decisões acerca de uma ou mais características da população em estudo. Por outro lado, muitos jogos de loterias também têm seus sorteios realizados a partir de processos cujas probabilidades de ganho são obtidas através do estudo de modelo de urnas. Um exemplo muito famoso no Brasil é a Mega-sena. No mais, a gama de problemas de probabilidade que pode ser pensada em termos de modelos de urnas é muito grande. Polya (1954) chegou a dizer que todo problema de probabilidade parece comparável a um problema de urnas contendo bolas. Nos dias atuais, além das evidentes aplicações na Física teórica e na Estatística, tem surgido uma imensidão de outras aplicações, das quais podemos destacar as aplicações nas áreas de tecnologia e mineração de dados. Para mais detalhes, veja Clarke, Fokoue e Zhang (2009).

Os problemas envolvendo modelos de urnas neste texto têm soluções baseadas em propriedades de sequências numéricas. Definimos, em cada problema, uma relação de recorrência, a qual é uma relação que determina cada termo de uma sequência como função de termos anteriores, e obtemos os resultados a partir de propriedades de sequências numéricas. Para que uma sequência seja descrita por uma equação de recorrência, é preciso estipular como se dá essa relação com termos anteriores 
e também um ou mais termos iniciais. Embora seja um assunto muito relevante e abrangente, em termos de ensino básico, poucas sequências são estudadas nessa etapa. Normalmente, trabalha-se com progressões aritméticas e geométricas. Outros tipos de sequência aparecem, quando muito, de maneira discreta e normalmente apenas como exemplos. Um exemplo clássico é a sequência de Fibonacci. Detalhes sobre sequências e progressões podem ser encontrados em Morgado, Wagner e Zani(2015) e Hazzan e Iezzi (2012). Para compreensão de problemas envolvendo modelos de urnas é importante estudar as técnicas básicas de contagem antes de atacar os modelos em si. Os conceitos básicos de contagem podem ser encontrados em Carvalho, Fernandez, Morgado e Pitombeira (2016), dos Santos, Melo e Murari (2008), Dante (2016), Almeida, Degenszajn, Dolce, Iezzi e Périgo (2016), Diniz e Smole (2016) e Hazzan(2013). Os problemas deste artigo podem ser encontrados em livros-textos de probabilidade como em Ross(2010) e Stirzaker (2003).

\section{Modelos}

Nesta seção apresentamos resultados acerca de alguns modelos de urnas cujas soluções são baseadas em propriedades de sequências numéricas. Os resultados são obtidos a partir da teoria básica de probabilidade. Basicamente evocamos a fórmula da probabilidade total para dois eventos (dada pela Proposição 1) e os resultados seguem a partir de propriedades de séries e sequências após obtenção de fórmulas de recorrência adequadas.

Proposição 1. (Fórmula da probabilidade total) Sejam A e B dois eventos aleatórios para o espaço amostral $\Omega$. Então,

$$
\mathbb{P}(\mathrm{A})=\mathbb{P}(\mathrm{B}) \mathbb{P}(\mathrm{A} \mid \mathrm{B})+\mathbb{P}\left(\mathrm{B}^{\mathrm{C}}\right) \mathbb{P}\left(\mathrm{A} \mid \mathrm{B}^{\mathrm{C}}\right) .
$$

Demonstração. Aqui, B e $\mathrm{B}^{\mathrm{C}}$ formam uma partição do espaço amostral $\Omega$ (Ver Figura 1).

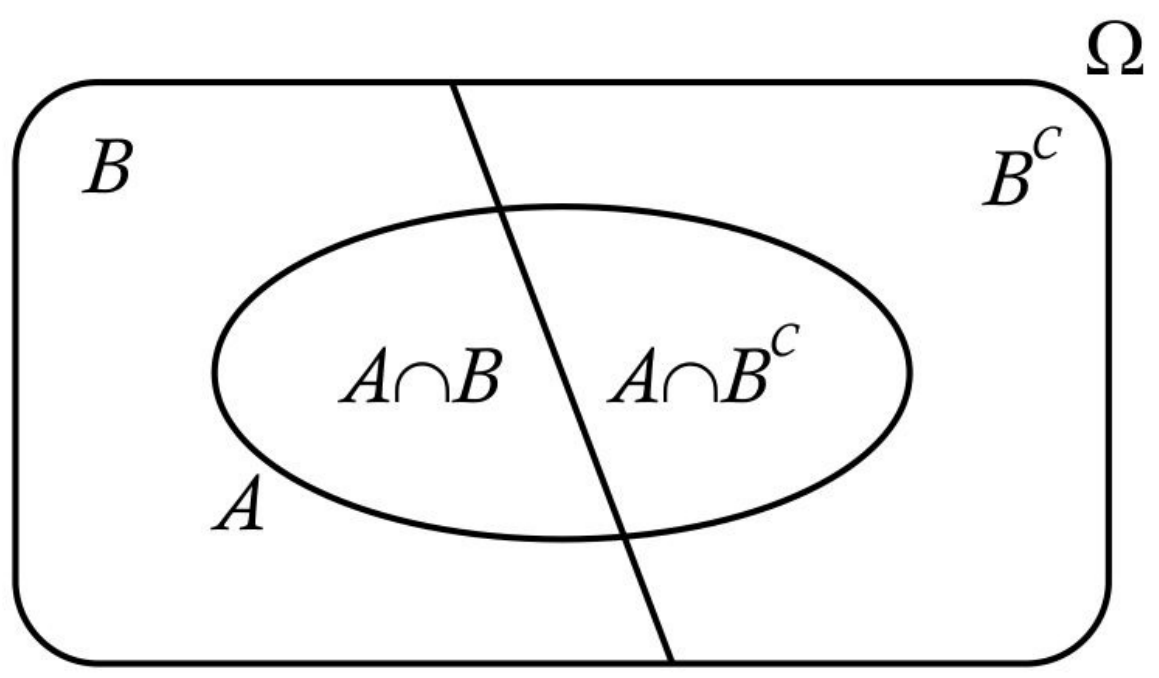

Figura 1: Partição do espaço amostral $\Omega$. 
Assim,

$$
\mathbb{P}(\mathrm{A})=\mathbb{P}(\mathrm{A} \cap \mathrm{B})+\mathbb{P}\left(\mathrm{A} \cap \mathrm{B}^{\mathrm{C}}\right) .
$$

Como $\mathbb{P}(\mathrm{A} \cap \mathrm{B})=\mathbb{P}(\mathrm{B}) \mathbb{P}(\mathrm{A} \mid \mathrm{B})$ e $\mathbb{P}\left(\mathrm{A} \cap \mathrm{B}^{\mathrm{C}}\right)=\mathbb{P}\left(\mathrm{B}^{\mathrm{C}}\right) \mathbb{P}\left(\mathrm{A} \mid \mathrm{B}^{\mathrm{C}}\right)$, segue o resultado.

A fórmula da probabilidade total pode ser estendida para qualquer quantidade finita ou, mais geralmente, enumerável de eventos formadores de uma partição do espaço amostral $\Omega$ (Veja Ross (2010)). Intuitivamente falando, usamos a fórmula da probabilidade total em experimentos envolvendo duas ou mais etapas para calcular probabilidade de um evento A, o qual não podemos obter o valor de forma direta. Esse resultado permite-nos, em algumas situações, obter indiretamente a probabilidade de um dado evento A, e, em outras situações, montar equações de recorrência para encontrar a probabilidade de um evento relativo à n-ésima etapa de um experimento composto (experimento com duas ou mais etapas). Neste trabalho, usaremos a fórmula da probabilidade total com esse intuito.

No que se segue, apresentaremos cinco modelos de urnas os quais exemplificam a modelagem de problemas probabilísticos via equações de recorrência.

Inicialmente, considere a seguinte dinâmica realizada com duas urnas. A urna I contém a 1 bolas azuis e $\mathrm{v}_{1}$ bolas vermelhas, enquanto que a urna II contém $\mathrm{a}_{2}$ bolas azuis e $\mathrm{v}_{2}$ bolas vermelhas. Bolas são extraídas sequencialmente das urnas e recolocadas de acordo com a seguinte regra: com probabilidade $\alpha$, retira-se inicialmente uma bola da urna I, e, com probabilidade $1-\alpha$, retira-se uma bola da urna II. As escolhas posteriores são então realizadas de acordo com o esquema de que sempre que uma bola azul for extraída (e devolvida), a próxima bola é sacada da mesma urna. Por outro lado, se a bola extraída (e devolvida) for vermelha, a próxima bola é retirada da outra urna.

Escreva

$$
\mathrm{p}_{1}=\frac{\mathrm{a}_{1}}{\mathrm{a}_{1}+\mathrm{v}_{1}} \text { e } \mathrm{p}_{2}=\frac{\mathrm{a}_{2}}{\mathrm{a}_{2}+\mathrm{v}_{2}}
$$

Aqui, $\mathrm{p}_{1}$ é a probabilidade de se extrair ao acaso uma bola azul da urna I. Analogamente, $\mathrm{p}_{2}$ é a probabilidade de se extrair ao acaso uma bola azul da urna II.

Teorema 1. Seja $\alpha_{\mathrm{n}}$ a probabilidade de que a n-ésima bola seja escolhida da urna I. Então,

$$
\alpha_{\mathrm{n}}=\frac{1-\mathrm{p}_{2}}{2-\mathrm{p}_{1}-\mathrm{p}_{2}}+\left[\alpha-\frac{\left(1-\mathrm{p}_{2}\right)}{2-\mathrm{p}_{1}-\mathrm{p}_{2}}\right]\left(\mathrm{p}_{1}+\mathrm{p}_{2}-1\right)^{\mathrm{n}-1}
$$

$e$

$$
\lim _{n \rightarrow \infty} \alpha_{n}=\frac{1-p_{2}}{2-p_{1}-p_{2}} .
$$

Seja $\beta_{\mathrm{n}}$ a probabilidade de que a n-ésima bola escolhida seja azul. Então,

$$
\beta_{\mathrm{n}}=\left(\mathrm{p}_{1}-\mathrm{p}_{2}\right)\left[\frac{1-\mathrm{p}_{2}}{2-\mathrm{p}_{1}-\mathrm{p}_{2}}+\left(\alpha-\frac{\left(1-\mathrm{p}_{2}\right)}{2-\mathrm{p}_{1}-\mathrm{p}_{2}}\right)\left(\mathrm{p}_{1}+\mathrm{p}_{2}-1\right)^{\mathrm{n}-1}\right]+\mathrm{p}_{2}
$$

$$
\lim _{n \rightarrow \infty} \beta_{n}=\frac{p_{1}-2 p_{1} p_{2}+p_{2}}{2-p_{1}-p_{2}}
$$


Demonstração. Defina $\mathrm{B}_{\mathrm{n}}$ como o evento onde a n-ésima bola sorteada vem da urna I. Logo, pela fórmula da probabilidade total,

$$
\mathbb{P}\left(B_{n+1}\right)=\mathbb{P}\left(B_{n}\right) \mathbb{P}\left(B_{n+1} \mid B_{n}\right)+\mathbb{P}\left(B_{n}^{C}\right) \mathbb{P}\left(B_{n+1} \mid B_{n}^{C}\right),
$$

então,

$$
\alpha_{\mathrm{n}+1}=\mathrm{p}_{1} \alpha_{\mathrm{n}}+\left(1-\mathrm{p}_{2}\right)\left(1-\alpha_{\mathrm{n}}\right)
$$

Daí,

$$
\alpha_{\mathrm{n}+1}=\left(\mathrm{p}_{1}+\mathrm{p}_{2}-1\right) \alpha_{\mathrm{n}}+\left(1-\mathrm{p}_{2}\right) .
$$

Escreva $\mathrm{c}=\mathrm{p}_{1}+\mathrm{p}_{2}-1$ e $\mathrm{s}=1-\mathrm{p}_{2}$. Então, fica $\alpha_{\mathrm{n}+1}=\mathrm{c} \alpha_{\mathrm{n}}+\mathrm{s}, \mathrm{n} \geq 1$ e $\alpha_{1}=\alpha$. Daí, temos

$$
\begin{aligned}
\alpha_{\mathrm{n}} & =\mathrm{c} \alpha_{\mathrm{n}-1}+\mathrm{s} \\
\mathrm{c} \alpha_{\mathrm{n}-1} & =\mathrm{c}^{2} \alpha_{\mathrm{n}-2}+\mathrm{sc} \\
\vdots & \\
\mathrm{c}^{\mathrm{n}-2} \alpha_{2} & =\mathrm{c}^{\mathrm{n}-1} \alpha_{1}+\mathrm{c}^{\mathrm{n}-2} \mathrm{~s} .
\end{aligned}
$$

Somando essas equações:

$$
\alpha_{\mathrm{n}}=\mathrm{c}^{\mathrm{n}-1} \alpha_{1}+\left(1+\mathrm{c}+\ldots+\mathrm{c}^{\mathrm{n}-2}\right) \mathrm{s} .
$$

Logo, usando a fórmula para soma de termos de uma progressão geométrica finita:

$$
\alpha_{\mathrm{n}}=\mathrm{c}^{\mathrm{n}-1} \alpha+\left(\frac{1-\mathrm{c}^{\mathrm{n}-1}}{1-\mathrm{c}}\right) \mathrm{s}
$$

e, assim,

$$
\alpha_{\mathrm{n}}=\frac{1-\mathrm{p}_{2}}{2-\mathrm{p}_{1}-\mathrm{p}_{2}}+\left[\alpha-\frac{\left(1-\mathrm{p}_{2}\right)}{2-\mathrm{p}_{1}-\mathrm{p}_{2}}\right]\left(\mathrm{p}_{1}+\mathrm{p}_{2}-1\right)^{\mathrm{n}-1}
$$

Tomando $\mathrm{n} \rightarrow \infty$

$$
\lim _{n \rightarrow \infty} \alpha_{n}=\lim _{n \rightarrow \infty}\left\{\frac{1-p_{2}}{2-p_{1}-p_{2}}+\left[\alpha-\frac{\left(1-p_{2}\right)}{2-p_{1}-p_{2}}\right]\left(p_{1}+p_{2}-1\right)^{n-1}\right\}=\frac{1-p_{2}}{2-p_{1}-p_{2}} .
$$

Agora, seja o evento $A_{n}$ onde a n-ésima bola sorteada é azul. Temos:

$$
\mathbb{P}\left(\mathrm{A}_{\mathrm{n}}\right)=\mathbb{P}\left(\mathrm{B}_{\mathrm{n}}\right) \mathbb{P}\left(\mathrm{A}_{\mathrm{n}} \mid \mathrm{B}_{\mathrm{n}}\right)+\mathbb{P}\left(\mathrm{B}_{\mathrm{n}}^{\mathrm{C}}\right) \mathbb{P}\left(\mathrm{A}_{\mathrm{n}} \mid \mathrm{B}_{\mathrm{n}}^{\mathrm{C}}\right),
$$

assim,

$$
\beta_{\mathrm{n}}=\mathrm{p}_{1} \alpha_{\mathrm{n}}+\mathrm{p}_{2}\left(1-\alpha_{\mathrm{n}}\right)=\left(\mathrm{p}_{1}-\mathrm{p}_{2}\right) \alpha_{\mathrm{n}}+\mathrm{p}_{2}
$$

Logo,

$$
\beta_{\mathrm{n}}=\left(\mathrm{p}_{1}-\mathrm{p}_{2}\right)\left[\frac{1-\mathrm{p}_{2}}{2-\mathrm{p}_{1}-\mathrm{p}_{2}}+\left(\alpha-\frac{\left(1-\mathrm{p}_{2}\right)}{2-\mathrm{p}_{1}-\mathrm{p}_{2}}\right)\left(\mathrm{p}_{1}+\mathrm{p}_{2}-1\right)^{\mathrm{n}-1}\right]+\mathrm{p}_{2}
$$

Tomando $\mathrm{n} \rightarrow \infty$

$$
\begin{gathered}
\lim _{n \rightarrow \infty} \beta_{n}=\lim _{n \rightarrow \infty}\left[\alpha_{n}\left(p_{1}-p_{2}\right)+p_{2}\right]=\left(p_{1}-p_{2}\right) \lim _{n \rightarrow \infty} \alpha_{n}+p_{2}=\left(p_{1}-p_{2}\right) \frac{1-p_{2}}{2-p_{1}-p_{2}}+p_{2} . \\
\lim _{n \rightarrow \infty} \beta_{n}=\frac{p_{1}-2 p_{1} p_{2}+p_{2}}{2-p_{1}-p_{2}} .
\end{gathered}
$$


Como aplicação do Teorema 1 considere o caso onde $\mathrm{a}_{1}=795, \mathrm{v}_{1}=5, \mathrm{a}_{2}=598$ e $\mathrm{v}_{2}=2$. Nesse caso, as duas urnas têm uma proporção muito grande de bolas azuis. Os valores das probabilidades $\alpha_{\mathrm{n}}$ e $\beta_{\mathrm{n}}$ bem como seus limites são:

$$
\begin{gathered}
\alpha_{\mathrm{n}}=\frac{8}{23}+\frac{7}{46}\left(\frac{2377}{2400}\right)^{\mathrm{n}-1}, \mathrm{n} \geq 1 \text { e } \lim _{\mathrm{n} \rightarrow \infty} \alpha_{\mathrm{n}}=\frac{8}{23}, \\
\beta_{\mathrm{n}}=\frac{229}{230}-\frac{49}{109342}\left(\frac{2377}{2400}\right)^{\mathrm{n}}, \mathrm{n} \geq 1 \text { e } \lim _{\mathrm{n} \rightarrow \infty} \beta_{\mathrm{n}}=\frac{229}{230} .
\end{gathered}
$$

Os gráficos a seguir apresentam as convergências das funções $\alpha_{\mathrm{n}}$ e $\beta_{\mathrm{n}}$, respectivamente.

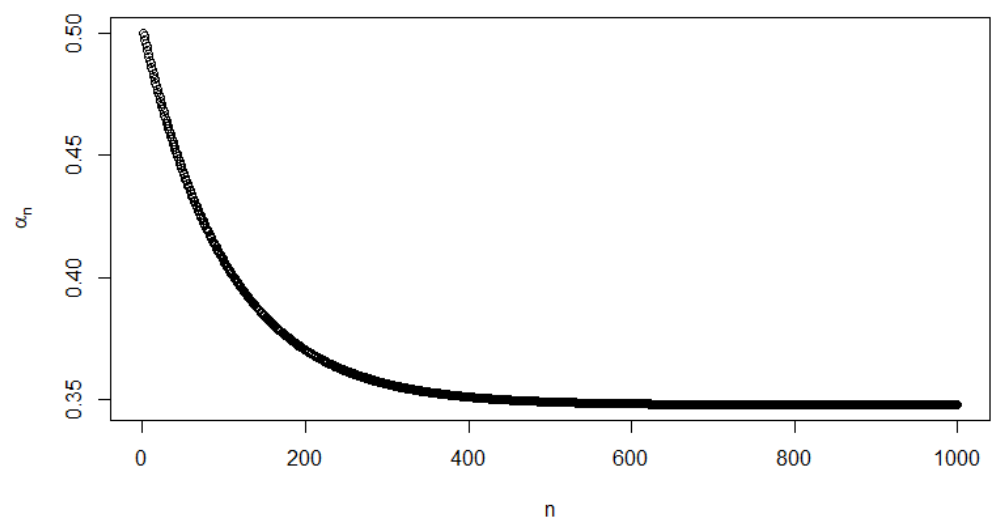

Figura 2: Probabilidade de que a n-ésima bola seja escolhida da urna I em função de n.

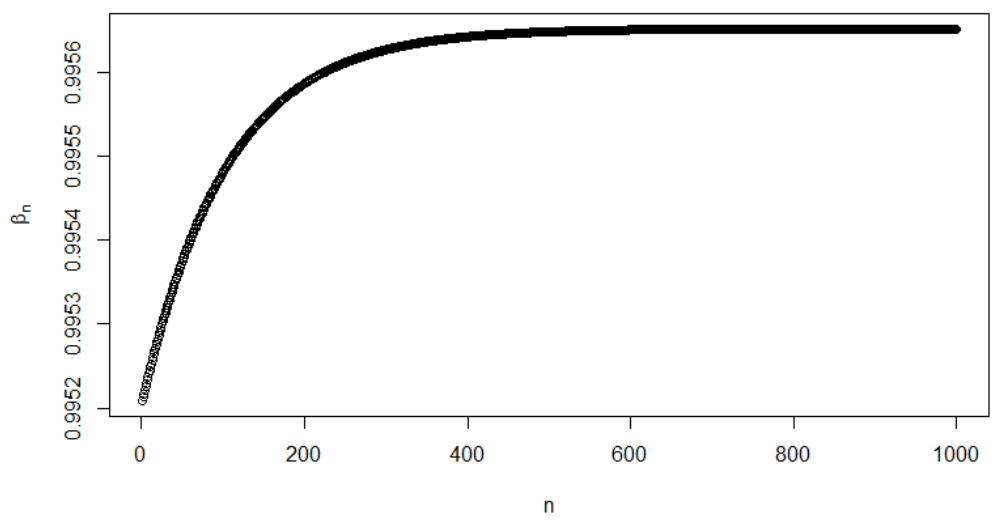

Figura 3: Probabilidade de que a n-ésima bola extraída seja azul em função de n. 
Agora, considere uma única urna contendo bolas diferindo unicamente pela cor. Inicialmente há na urna a bolas azuis e v bolas vermelhas, onde a > v. Bolas são extraídas uma a uma ao acaso e sem reposição da urna até que essa fique vazia.

Seja $\mathrm{p}_{\mathrm{a}, \mathrm{v}}$ a probabilidade de que, ao longo da contagem das bolas extraídas, o número de bolas azuis sempre seja maior do que o número de bolas vermelhas. O teorema a seguir é conhecido como Teorema de Ballot.

Teorema 2. Temos

$$
\mathrm{p}_{\mathrm{a}, \mathrm{v}}=\frac{\mathrm{a}-\mathrm{v}}{\mathrm{a}+\mathrm{v}}
$$

Demonstração. Defina os eventos A onde a última bola retirada é azul, e B onde ao longo da contagem das bolas extraídas, o número de bolas azuis sempre é maior do que o número de bolas vermelhas. Pela fórmula da probabilidade total:

$$
\begin{aligned}
\mathbb{P}(\mathrm{B}) & =\mathbb{P}(\mathrm{A}) \mathbb{P}(\mathrm{B} \mid \mathrm{A})+\mathbb{P}\left(\mathrm{A}^{\mathrm{C}}\right) \mathbb{P}\left(\mathrm{B} \mid \mathrm{A}^{\mathrm{C}}\right) \\
\mathrm{p}_{\mathrm{a}, \mathrm{v}} & =\frac{\mathrm{a}}{(\mathrm{a}+\mathrm{v})} \mathrm{p}_{\mathrm{a}-1, \mathrm{v}}+\frac{\mathrm{v}}{(\mathrm{a}+\mathrm{v})} \mathrm{p}_{\mathrm{a}, \mathrm{v}-1} .
\end{aligned}
$$

Faremos a prova por indução. Primeiro supomos que para $\mathrm{a}+\mathrm{v}=\mathrm{k}-1$ o resultado vale, isto é,

$$
\mathrm{p}(\mathrm{k}-1)=\mathrm{p}_{\mathrm{a}, \mathrm{v}}=\frac{\mathrm{a}-\mathrm{v}}{\mathrm{a}+\mathrm{v}}
$$

Logo,

$$
\begin{aligned}
& \mathrm{p}(\mathrm{k})=\frac{\mathrm{a}}{(\mathrm{a}+\mathrm{v})} \mathrm{p}_{\mathrm{a}-1, \mathrm{v}}+\frac{\mathrm{v}}{(\mathrm{a}+\mathrm{v})} \mathrm{p}_{\mathrm{a}, \mathrm{v}-1}=\frac{\mathrm{a}}{(\mathrm{a}+\mathrm{v})} \frac{\mathrm{a}-1-\mathrm{v}}{(\mathrm{a}-1+\mathrm{v})}+\frac{\mathrm{v}}{(\mathrm{a}+\mathrm{v})} \frac{(\mathrm{a}-(\mathrm{v}-1))}{(\mathrm{a}+\mathrm{v}-1)} \\
& \mathrm{p}(\mathrm{k})=\frac{\mathrm{a}}{(\mathrm{a}+\mathrm{v})} \frac{(\mathrm{a}-1-\mathrm{v})}{(\mathrm{a}-1+\mathrm{v})}+\frac{\mathrm{v}}{(\mathrm{a}+\mathrm{v})} \frac{(\mathrm{a}-\mathrm{v}+1)}{(\mathrm{a}+\mathrm{v}-1)}=\frac{\mathrm{a}^{2}-\mathrm{a}-\mathrm{av}+\mathrm{av}-\mathrm{v}^{2}+\mathrm{v}}{(\mathrm{a}+\mathrm{v})(\mathrm{a}-1+\mathrm{v})} \\
& \mathrm{p}(\mathrm{k})=\frac{(\mathrm{a}-\mathrm{v})(\mathrm{a}+\mathrm{v})-(\mathrm{a}-\mathrm{v})}{(\mathrm{a}+\mathrm{v})(\mathrm{a}-1+\mathrm{v})}=\frac{\mathrm{a}-\mathrm{v}}{\mathrm{a}+\mathrm{v}} .
\end{aligned}
$$

Acabamos de provar uma conjectura, mas como chegar a ela? A ideia é obter primeiro valores de $\mathrm{p}_{\mathrm{a}, \mathrm{v}}$ para a e v pequenos. Com algum esforço obtém-se:

$$
\mathrm{p}_{2,1}=\frac{1}{3}, \mathrm{p}_{3,1}=\frac{2}{4}, \mathrm{p}_{3,2}=\frac{1}{5}, \mathrm{p}_{4,1}=\frac{3}{5}, \mathrm{p}_{4,2}=\frac{2}{6}, \text { etc }
$$

Daí, fixamos v e podemos obter

$$
\mathrm{p}_{\mathrm{a}, 1}=\frac{\mathrm{a}-1}{\mathrm{a}+1}, \mathrm{p}_{\mathrm{a}, 2}=\frac{\mathrm{a}-2}{\mathrm{a}+2}
$$

Com alguma imaginação conjecturamos $\mathrm{p}_{\mathrm{a}, \mathrm{v}}=\frac{\mathrm{a}-\mathrm{v}}{\mathrm{a}+\mathrm{v}}$.

A tabela a seguir apresenta as probabilidades $\mathrm{p}_{\mathrm{a}, \mathrm{v}}$ de que, ao longo da contagem das bolas extraídas, o número de bolas azuis seja sempre maior do que o número de bolas vermelhas em função do número de bolas azuis (a) e vermelhas (v). 


\begin{tabular}{|c|c|c|}
\hline $\mathrm{a}$ & $\mathrm{v}$ & $\mathrm{p}_{\mathrm{a}, \mathrm{v}}$ \\
\hline \hline 2 & 1 & 0.3333333 \\
\hline 3 & 1 & 0.5000000 \\
\hline 4 & 1 & 0.6000000 \\
\hline 5 & 1 & 0.6666667 \\
\hline 3 & 2 & 0.2000000 \\
\hline 4 & 2 & 0.3333333 \\
\hline 5 & 2 & 0.4285714 \\
\hline 4 & 3 & 0.1428571 \\
\hline 5 & 3 & 0.2500000 \\
\hline 5 & 4 & 0.1111111 \\
\hline
\end{tabular}

Tabela 1: Probabilidades $\mathrm{p}_{\mathrm{a}, \mathrm{v}}$ de que, ao longo da contagem das bolas extraídas, o número de bolas azuis seja sempre maior do que o número de bolas vermelhas em função do número de bolas azuis (a) e vermelhas (v).

Cabe ressaltar que fixado o número de bolas vermelhas, o valor da probabilidade $\mathrm{p}_{\mathrm{a}, \mathrm{v}}$ aumenta em função da quantidade de bolas azuis a mais que bolas vermelhas. De fato, podemos escrever

$$
\mathrm{h}(\mathrm{a}, \mathrm{v}):=\mathrm{p}_{\mathrm{a}, \mathrm{v}}=1-\frac{2 \mathrm{v}}{\mathrm{a}+\mathrm{v}} .
$$

Para ilustrar a evolução dessas probabilidades em função do número de bolas azuis a, fixamos v $=3$ bolas vermelhas. Nesse caso, $h(a, 3)=1-\frac{6}{a+3}$.

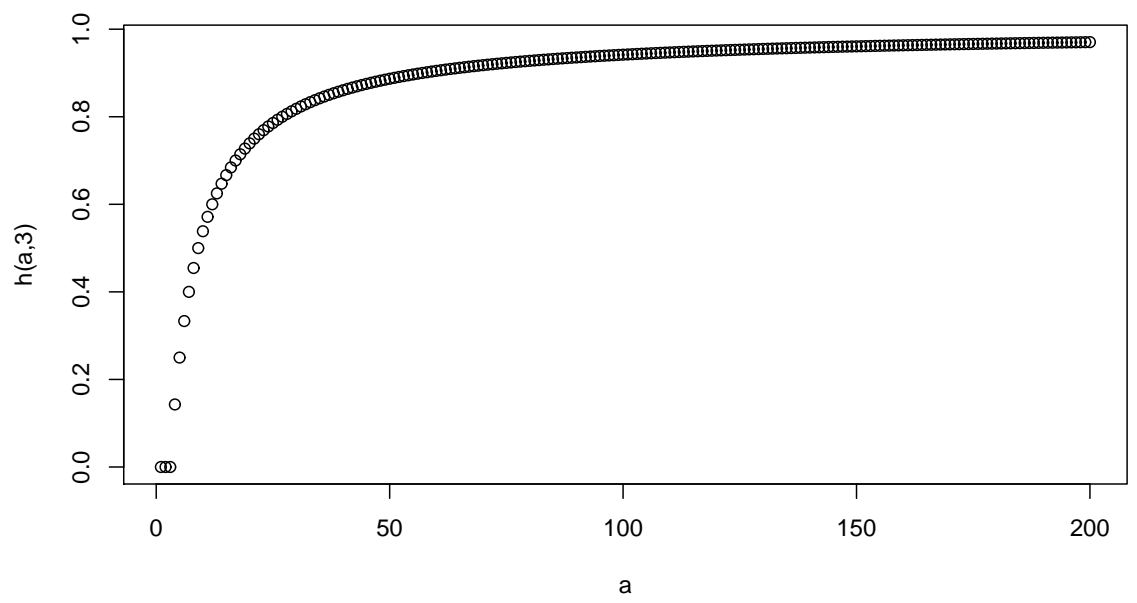

Figura 4: Evolução das Probabilidades $h(a, v)=p_{a, v}$ para $v=3$.

A seguir considere uma urna contendo bolas diferindo unicamente pela cor. Inicialmente há na urna 
a bolas azuis e v bolas vermelhas. Dois jogadores, $\mathrm{J}_{\mathrm{A}}$ e $\mathrm{J}_{\mathrm{V}}$, apostam nos resultados de sucessivas extrações dessa urna. Em cada extração, se der bola azul, $\mathrm{J}_{\mathrm{A}}$ ganha 1 unidade monetária de $\mathrm{J}_{\mathrm{V}}$. Se der bola vermelha, $\mathrm{J}_{\mathrm{V}}$ ganha uma unidade monetária de $\mathrm{J}_{\mathrm{A}}$. Eles continuam jogando indefinidamente a menos que um deles fique sem dinheiro. Após cada extração, a bola extraída é devolvida.

Teorema 3. Seja $\mathrm{p}_{\mathrm{i}}$ a probabilidade que $\mathrm{J}_{\mathrm{A}}$ termine com todo o dinheiro, tendo começado com $\mathrm{i}$ unidades monetárias e $\mathrm{J}_{\mathrm{V}}$ com $\mathrm{N}-\mathrm{i}$ unidades. Temos $\mathrm{v} \neq \mathrm{a}$

$$
\mathrm{p}_{\mathrm{i}}=\frac{1-\left(\frac{\mathrm{v}}{\mathrm{a}}\right)^{\mathrm{i}}}{1-\left(\frac{\mathrm{v}}{\mathrm{a}}\right)^{\mathrm{N}}}
$$

e se $\mathrm{v}=\mathrm{a}, \mathrm{p}_{\mathrm{i}}=\frac{\mathrm{i}}{\mathrm{N}}$. Seja $\mathrm{q}_{\mathrm{i}}$ a probabilidade de que $\mathrm{J}_{\mathrm{V}}$ termine com todo o dinheiro, quando $\mathrm{J}_{\mathrm{A}}$ começa com $\mathrm{i}$ unidades monetárias e $\mathrm{J}_{\mathrm{V}}$ com $\mathrm{N}-\mathrm{i}$ unidades temos para $\mathrm{a} \neq \mathrm{v}$ :

$$
\mathrm{q}_{i}=\frac{1-\left(\frac{\mathrm{a}}{\mathrm{v}}\right)^{\mathrm{N}-\mathrm{i}}}{1-\left(\frac{\mathrm{a}}{\mathrm{v}}\right)^{\mathrm{N}}}
$$

e $\mathrm{q}_{\mathrm{i}}=\frac{\mathrm{N}-\mathrm{i}}{\mathrm{N}}$ para $\mathrm{a}=\mathrm{v}$. Por fim, se $\mathrm{r}_{\mathrm{i}}$ é a probabilidade de que o jogo nunca termina quando $\mathrm{J}_{\mathrm{A}}$ começa com $\mathrm{i}$ unidades e $\mathrm{J}_{\mathrm{V}}$ com $\mathrm{N}$ - i unidades, então, $\mathrm{r}_{\mathrm{i}}=0$.

Demonstração. Suponha que A seja o evento onde $\mathrm{J}_{\mathrm{A}}$ termina com todo o dinheiro, tendo começado com i unidades e $\mathrm{J}_{\mathrm{V}}$ com $\mathrm{N}-\mathrm{i}$ unidades. Escreva $\mathrm{p}_{\mathrm{i}}=\mathbb{P}(\mathrm{A})$. Defina $\mathrm{Z}$ como o evento em que a extração da primeira bola resulta em bola azul. Condicionado na extração da primeira bola, temos:

$$
\mathrm{p}_{\mathrm{i}}=\mathbb{P}(\mathrm{A})=\mathbb{P}(\mathrm{Z}) \mathbb{P}(\mathrm{A} \mid \mathrm{Z})+\mathbb{P}\left(\mathrm{Z}^{\mathrm{C}}\right) \mathbb{P}\left(\mathrm{A} \mid \mathrm{Z}^{\mathrm{C}}\right)=\frac{\mathrm{a}}{\mathrm{a}+\mathrm{v}} \mathbb{P}(\mathrm{A} \mid \mathrm{Z})+\frac{\mathrm{v}}{\mathrm{a}+\mathrm{v}} \mathbb{P}\left(\mathrm{A} \mid \mathrm{Z}^{\mathrm{C}}\right)
$$

Note que, uma vez que a primeira extração resulta em bola azul, após a primeira aposta $\mathrm{J}_{\mathrm{A}}$ fica com $\mathrm{i}+1$ unidades enquanto $\mathrm{J}_{\mathrm{V}}$ fica com $\mathrm{N}-(\mathrm{i}+1)$. Por outro lado, se a extração resultar em bola vermelha, ao fim da primeira aposta, $\mathrm{J}_{\mathrm{A}}$ fica com $\mathrm{i}-1$ unidades, enquanto $\mathrm{J}_{\mathrm{V}}$ fica com $\mathrm{N}-(\mathrm{i}-1)$. Como os resultados das apostas sucessivas são independentes, temos

$$
\mathbb{P}(\mathrm{A} \mid \mathrm{Z})=\mathrm{p}_{\mathrm{i}+1} \text { e } \mathbb{P}\left(\mathrm{A} \mid \mathrm{Z}^{\mathrm{C}}\right)=\mathrm{p}_{\mathrm{i}-1}
$$

Logo, podemos escrever

$$
\mathrm{p}_{\mathrm{i}}=\frac{\mathrm{a}}{\mathrm{a}+\mathrm{v}} \mathrm{p}_{\mathrm{i}+1}+\frac{\mathrm{v}}{\mathrm{a}+\mathrm{v}} \mathrm{p}_{\mathrm{i}-1}, \mathrm{i}=1,2, \ldots, \mathrm{N}-1 .
$$

Temos ainda as condições de contorno $\mathrm{p}_{0}=0$ e $\mathrm{p}_{\mathrm{N}}=1$.

Assim,

$$
\mathrm{p}_{\mathrm{i}+1}-\mathrm{p}_{\mathrm{i}}=\frac{\mathrm{v}}{\mathrm{a}}\left(\mathrm{p}_{\mathrm{i}}-\mathrm{p}_{\mathrm{i}-1}\right), \mathrm{i}=1,2, \ldots, \mathrm{N}-1 .
$$


Logo, como $\mathrm{p}_{0}=0$ :

$$
\begin{aligned}
\mathrm{p}_{2}-\mathrm{p}_{1} & =\frac{\mathrm{v}}{\mathrm{a}}\left(\mathrm{p}_{1}-\mathrm{p}_{0}\right)=\frac{\mathrm{v}}{\mathrm{a}} \\
\mathrm{p}_{3}-\mathrm{p}_{2} & =\frac{\mathrm{v}}{\mathrm{a}}\left(\mathrm{p}_{2}-\mathrm{p}_{1}\right)=\left(\frac{\mathrm{v}}{\mathrm{a}}\right)^{2} \mathrm{p}_{1} \\
\vdots & \\
\mathrm{p}_{\mathrm{i}}-\mathrm{p}_{\mathrm{i}-1} & =\frac{\mathrm{v}}{\mathrm{a}}\left(\mathrm{p}_{\mathrm{i}-1}-\mathrm{p}_{\mathrm{i}-2}\right)=\left(\frac{\mathrm{v}}{\mathrm{a}}\right)^{\mathrm{i}-1} \mathrm{p}_{1} \\
\vdots & \\
\mathrm{p}_{\mathrm{N}}-\mathrm{p}_{\mathrm{N}-1} & =\frac{\mathrm{v}}{\mathrm{a}}\left(\mathrm{p}_{\mathrm{N}-1}-\mathrm{p}_{\mathrm{N}-2}\right)=\left(\frac{\mathrm{v}}{\mathrm{a}}\right)^{\mathrm{N}-1} \mathrm{p}_{1} .
\end{aligned}
$$

Somando as i-1 primeiras equações,

$$
\mathrm{p}_{\mathrm{i}}-\mathrm{p}_{1}=\mathrm{p}_{1}\left[\left(\frac{\mathrm{v}}{\mathrm{a}}\right)+\left(\frac{\mathrm{v}}{\mathrm{a}}\right)^{2}+\cdots+\left(\frac{\mathrm{v}}{\mathrm{a}}\right)^{\mathrm{i}-1} \cdot\right]
$$

Assim, se $\mathrm{a} \neq \mathrm{v}$, aplicamos a soma dos termos de uma progressão geométrica finita e obtemos

$$
\mathrm{p}_{\mathrm{i}}=\left[\frac{1-\left(\frac{\mathrm{v}}{\mathrm{a}}\right)^{\mathrm{i}}}{1-\frac{\mathrm{v}}{\mathrm{a}}}\right] \mathrm{p}_{1} .
$$

Como $\mathrm{p}_{\mathrm{N}}=1$, temos

$$
\mathrm{p}_{1}=\frac{1-\frac{\mathrm{v}}{\mathrm{a}}}{1-\left(\frac{\mathrm{v}}{\mathrm{a}}\right)^{\mathrm{N}}}
$$

e, assim,

$$
\mathrm{p}_{\mathrm{i}}=\frac{1-\left(\frac{\mathrm{v}}{\mathrm{a}}\right)^{\mathrm{i}}}{1-\left(\frac{\mathrm{v}}{\mathrm{a}}\right)^{\mathrm{N}}} .
$$

Por outro lado, se $\mathrm{a}=\mathrm{v}, \mathrm{p}_{\mathrm{i}}=\mathrm{ip}_{1}$, donde $\mathrm{p}_{1}=\frac{1}{\mathrm{~N}}$, e, assim, $\mathrm{p}_{\mathrm{i}}=\frac{\mathrm{i}}{\mathrm{N}}$.

De modo inteiramente similar, se escrevermos $\mathrm{q}_{\mathrm{i}}$ como a probabilidade de que $\mathrm{J}_{\mathrm{V}}$ termine com todo o dinheiro, quando $\mathrm{J}_{\mathrm{A}}$ começa com i unidades e $\mathrm{J}_{\mathrm{V}}$ com $\mathrm{N}$ - i unidades, obtemos para a $\neq \mathrm{v}$ :

$$
\mathrm{q}_{\mathrm{i}}=\frac{1-\left(\frac{\mathrm{a}}{\mathrm{v}}\right)^{\mathrm{N}-\mathrm{i}}}{1-\left(\frac{\mathrm{a}}{\mathrm{v}}\right)^{\mathrm{N}}},
$$

e $q_{i}=\frac{N-i}{N}$ para $a=v$. Por fim, note que existem três possibilidades: $J_{A}$ vence, $J_{V}$ vence ou o jogo continua indefinidamente sem que ninguém vença. Seja $r_{i}$ a probabilidade de que o jogo nunca termine. Para $a \neq r$, de $p_{i}+q_{i}+r_{i}=1$ temos $r_{i}=0$, pois

$$
p_{i}+q_{i}=\frac{1-\left(\frac{v}{a}\right)^{i}}{1-\left(\frac{v}{a}\right)^{N}}+\frac{1-\left(\frac{a}{v}\right)^{N-i}}{1-\left(\frac{a}{v}\right)^{N}}=1 .
$$

Para $\mathrm{a}=\mathrm{r}$ :

$$
\mathrm{p}_{\mathrm{i}}+\mathrm{q}_{\mathrm{i}}=\frac{\mathrm{i}}{\mathrm{N}}+\frac{\mathrm{N}-\mathrm{i}}{\mathrm{N}}=1
$$

E, nesse caso, também, $r_{i}=0$. 
O Teorema 3 apresenta uma propriedade muito interessante para o caso onde $\mathrm{v}<\mathrm{a}$. Mesmo que o jogador $\mathrm{J}_{\mathrm{V}}$ inicie com uma quantidade muito grande de dinheiro, ele tem chance de sair derrotado da disputa e perder todo seu dinheiro. De fato, se $\mathrm{v}<\mathrm{a}$, fazendo $\mathrm{N} \rightarrow \infty$ temos:

$$
\begin{gathered}
\lim _{N \rightarrow \infty} p_{i}=\lim _{N \rightarrow \infty} \frac{1-\left(\frac{v}{a}\right)^{i}}{1-\left(\frac{v}{a}\right)^{N}}=1-\left(\frac{v}{a}\right)^{i} e \\
\lim _{N \rightarrow \infty} q_{i}=\lim _{N \rightarrow \infty} \frac{1-\left(\frac{a}{v}\right)^{N-i}}{1-\left(\frac{a}{v}\right)^{N}}=\left(\frac{v}{a}\right)^{i} .
\end{gathered}
$$

Agora, considere uma urna contendo bolas diferindo unicamente pela cor. Inicialmente há na urna a bolas azuis e v bolas vermelhas. Bolas são extraídas uma a uma ao acaso e com reposição.

Teorema 4. Seja $\mathrm{p}_{\mathrm{n}}$ a probabilidade de que em $\mathrm{n}$ extrações saiam um número par de bolas azuis. Então,

$$
\mathrm{p}_{\mathrm{n}}=\frac{1+\left(\frac{\mathrm{v}-\mathrm{a}}{\mathrm{v}+\mathrm{a}}\right)^{\mathrm{n}}}{2}, \mathrm{n}=1,2, \ldots
$$

Além disso,

$$
\lim _{n \rightarrow \infty} p_{n}=\frac{1}{2}
$$

Demonstração. Defina A o evento em que a primeira retirada resulta em bola azul e B o evento o qual em $n$ extrações saia um número par de bolas azuis. Temos $p_{n}=\mathbb{P}(B)$. Pela fórmula da probabilidade total temos

$$
\mathbb{P}(\mathrm{B})=\mathbb{P}(\mathrm{A}) \mathbb{P}(\mathrm{B} \mid \mathrm{A})+\mathbb{P}\left(\mathrm{A}^{\mathrm{C}}\right) \mathbb{P}\left(\mathrm{B} \mid \mathrm{A}^{\mathrm{C}}\right)
$$

Note que $\mathbb{P}(B \mid A)=1-p_{n-1}$. De fato, para $B$ ocorrer, dado que a primeira retirada resultou em bola azul, é preciso que nas próximas $\mathrm{n}-1$ extrações saia um número ímpar de bolas azuis, evento cuja probabilidade é $1-\mathrm{p}_{\mathrm{n}-1}$. Por outro lado, $\mathbb{P}\left(\mathrm{B} \mid \mathrm{A}^{\mathrm{C}}\right)=\mathrm{p}_{\mathrm{n}-1}$ pois para $\mathrm{B}$ ocorrer, dado que a primeira retirada resultou em bola vermelha, é preciso que nas próximas $n-1$ extrações saia um número par de bolas azuis, evento cuja probabilidade é $\mathrm{p}_{\mathrm{n}-1}$. Logo,

$$
\begin{aligned}
& \mathrm{p}_{\mathrm{n}}=\frac{\mathrm{a}}{\mathrm{v}+\mathrm{a}}\left(1-\mathrm{p}_{\mathrm{n}-1}\right)+\frac{\mathrm{v}}{\mathrm{v}+\mathrm{a}} \mathrm{p}_{\mathrm{n}-1}, \\
& \mathrm{p}_{\mathrm{n}}=\frac{\mathrm{a}}{(\mathrm{v}+\mathrm{a})}+\frac{(\mathrm{v}-\mathrm{a})}{(\mathrm{v}+\mathrm{a})} \mathrm{p}_{\mathrm{n}-1} .
\end{aligned}
$$

Escreva $c=\frac{a}{v+a}$ e $b=\frac{v-a}{v+a}$

$$
\begin{aligned}
p_{n} & =c+b p_{n-1} \\
b \cdot p_{n-1} & =b c+b^{2} p_{n-2} \\
b^{2} \cdot p_{n-2} & =b^{2} c+b^{3} p_{n-3} \\
\vdots & \\
b^{n-2} \cdot p_{2} & =b^{n-2} c+b^{n-1} p_{1} .
\end{aligned}
$$


Somando essas equações

$$
\mathrm{p}_{\mathrm{n}}=\mathrm{c}+\mathrm{cb}+\mathrm{cb} \mathrm{b}^{2}+\ldots+\mathrm{cb}^{\mathrm{n}-2}+\mathrm{b}^{\mathrm{n}-1} \mathrm{p}_{1}
$$

Aplicando a soma dos termos de uma progressão geométrica finita

$$
p_{n}=c\left(\frac{1-b^{n-1}}{1-b}\right)+b^{n-1} p_{1} \text {. }
$$

Como $\mathrm{p}_{1}=\frac{\mathrm{v}}{\mathrm{v}+\mathrm{a}}$

$$
\mathrm{p}_{\mathrm{n}}=\frac{1+\left(\frac{\mathrm{v}-\mathrm{a}}{\mathrm{v}+\mathrm{a}}\right)^{\mathrm{n}}}{2}, \mathrm{n}=1,2, \ldots
$$

Por fim,

$$
\lim _{n \rightarrow \infty} p_{n}=\lim _{n \rightarrow \infty} \frac{1}{2}+\frac{1}{2}\left(\frac{v-a}{v+a}\right)^{n}=\frac{1}{2}+\frac{1}{2} \lim _{n \rightarrow \infty}\left(\frac{v-a}{v+a}\right)^{n}=\frac{1}{2}
$$

Para exemplificar o resultado do Teorema 4 considere o caso o qual temos a $=995$ bolas azuis e $\mathrm{v}=5$ bolas vermelhas. O gráfico a seguir mostra o comportamento da função $\mathrm{p}(\mathrm{n})=\mathrm{p}_{\mathrm{n}}$ que dá a probabilidade de que em n extrações saia um número par de bolas azuis em função do número de extrações n. Nesse caso,

$$
\mathrm{p}(\mathrm{n})=\frac{1}{2}+\frac{1}{2}\left(\frac{-99}{100}\right)^{\mathrm{n}}, \mathrm{n} \geq 1 .
$$

Note que a função $\mathrm{p}(\mathrm{n})$ assume valores maiores que $\frac{1}{2}$ para $\mathrm{n}$ par e valores menores que $\frac{1}{2}$ para $n$ ímpar. Essa interessante propriedade vale em geral para todos os casos em que o número de bolas azuis é maior que o número de bolas vermelhas. Por outro lado, se o número de bolas vermelhas é maior que o número de bolas azuis, $\mathrm{p}(\mathrm{n})$ é uma função decrescente que converge para $\frac{1}{2}$.

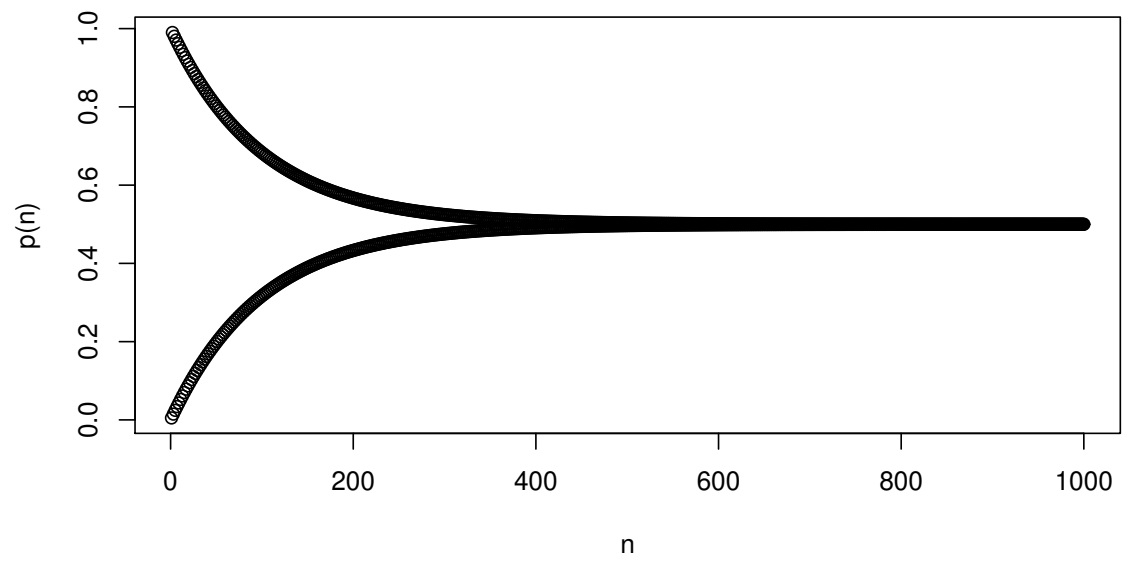

Figura 5: Evolução das Probabilidades $\mathrm{h}(\mathrm{a}, \mathrm{v})=\mathrm{p}_{\mathrm{a}, \mathrm{v}}$ para $\mathrm{v}=3$. 
Por fim, considere uma sequência de urnas numeradas de 1 até $n$ tal que a urna de número i $(\mathrm{i}=1,2, \ldots, \mathrm{n})$ possui $2 \mathrm{i}$ bolas azuis e 1 bola vermelha. Uma bola é extraída ao acaso de cada urna.

Teorema 5. Seja $\mathrm{p}_{\mathrm{n}}$ a probabilidade de que o número de bolas vermelhas extraídas seja ímpar. Então,

$$
\mathrm{p}_{\mathrm{n}}=\frac{\mathrm{n}}{2 \mathrm{n}+1}, \mathrm{n}=1,2, \ldots
$$

Demonstração. Defina A o evento onde a bola retirada na urna n é azul e B o evento onde nas $n$ extrações saia um número ímpar de bolas vermelhas. Temos $p_{n}=\mathbb{P}(B)$. Pela fórmula da probabilidade total temos

$$
\mathbb{P}(\mathrm{B})=\mathbb{P}(\mathrm{A}) \mathbb{P}(\mathrm{B} \mid \mathrm{A})+\mathbb{P}\left(\mathrm{A}^{\mathrm{C}}\right) \mathbb{P}\left(\mathrm{B} \mid \mathrm{A}^{\mathrm{C}}\right)
$$

Note que $\mathbb{P}\left(B \mid A^{C}\right)=1-p_{n-1}$. De fato, para $B$ ocorrer, dado que a retirada na n-ésima urna resultou em bola vermelha, é preciso que nas próximas $n-1$ extrações anteriores tenha saído um número par de bolas vermelhas, evento cuja probabilidade é $1-\mathrm{p}_{\mathrm{n}-1}$. Por outro lado, $\mathbb{P}(\mathrm{B} \mid \mathrm{A})=\mathrm{p}_{\mathrm{n}-1}$, pois para $\mathrm{B}$ ocorrer, dado que a retirada na n-ésima urna resultou em bola azul, é preciso que nas $n-1$ extrações anteriores tenha saído um número ímpar de bolas vermelhas, evento cuja probabilidade é $\mathrm{p}_{\mathrm{n}-1}$. Logo,

$$
\mathrm{p}_{\mathrm{n}}=\left(\frac{2 \mathrm{n}}{2 \mathrm{n}+1}\right) \mathrm{p}_{\mathrm{n}-1}+\left(\frac{1}{2 \mathrm{n}+1}\right)\left(1-\mathrm{p}_{\mathrm{n}-1}\right)
$$

E, assim,

$$
(2 \mathrm{n}+1) \mathrm{p}_{\mathrm{n}}-(2 \mathrm{n}-1) \mathrm{p}_{\mathrm{n}-1}=1 .
$$

Daí,

$$
\begin{aligned}
(2 \mathrm{n}+1) \mathrm{p}_{\mathrm{n}}-(2 \mathrm{n}-1) \mathrm{p}_{\mathrm{n}-1} & =1 \\
(2 \mathrm{n}-1) \mathrm{p}_{\mathrm{n}-1}-(2 \mathrm{n}-3) \mathrm{p}_{\mathrm{n}-2} & =1 \\
(2 \mathrm{n}-3) \mathrm{p}_{\mathrm{n}-2}-(2 \mathrm{n}-5) \mathrm{p}_{\mathrm{n}-3} & =1 \\
\vdots & \\
5 \mathrm{p}_{2}-3 \mathrm{p}_{1} & =1 .
\end{aligned}
$$

Como $\mathrm{p}_{1}=\frac{1}{3}$, somando essas $\mathrm{n}-1$ equações obtemos

$$
\mathrm{p}_{\mathrm{n}}=\frac{\mathrm{n}}{2 \mathrm{n}+1}, \mathrm{n}=1,2, \ldots
$$

Uma consequência do Teorema 5 é que a probabilidade de que o número de bolas vermelhas extraídas seja ímpar converge rapidamente para $\frac{1}{2}$.

\section{Considerações Finais}

Este trabalho apresenta modelos de simples compreensão com resultados bastante interessantes. Ele pode servir de motivação para estudantes que queiram se dedicar ao estudo da teoria de probabilidade. Modelos de urnas fornecem problemas de fácil entendimento. As abordagens estão 
relacionadas com problemas presentes no nosso cotidiano e fornecem base para cálculos probabilísticos envolvendo loterias bem como problemas associados à amostragem. A abordagem apresentada por nós torna-se interessante também por associar cálculo probabilístico ao uso de propriedades de sequências numéricas. Assim, mostramos uma das muitas relevantes aplicações do estudo de sequências e séries.

\section{Referências}

[1] Almeida, N., Degenszajn, D., Dolce, O., Iezzi, G., Périgo, R.; Matemática: ciência e aplicações, Saraiva, Volume 2, nona edição, 2016.

[2] Carvalho, P.C.P.,Fernandez, P., Morgado, A.C.O., Pitombeira, J.B.; Análise Combinatória e Probabilidade. SBM, décima edição, 2016.

[3] Charalambides, A. C.; Combinatorial Methods in Discrete Distributions. Wiley, primeira edição, 2005.

[4] Clarke, B.; Fokoue, E.; Zhang, H. H.; Principles and theory for data mining and machine learning. Springer Science \& Business Media, 2009.

[5] Dante, L.R.; Matemática: contexto $\&$ aplicações: ensino médio, Ática, Volume 2, terceira edição, 2016.

[6] Diniz, M.I., Smole, K.S. Matemática para compreender o mundo, Saraiva, Volume 2, primeira edição, 2016.

[7] Hazzan, S. Fundamentos de matemática elementar: combinatória e probabilidade. Atual editora, Volume 5, 2013.

[8] Heubeck, K.; Urnenmodelle und ihre Anwendung in der Versicherungsmathematik, BI. Deut. Ges. Versicher rngsmuth., 11 (3), 37 1-429, 1974.

[9] Hazzan, S.; Iezzi,G.; Fundamentos de matemática elementar: Sequências, matrizes, determinantes e sistemas. Atual editora, Volume 4, 2012.

[10] Johnson, N.L; Kotz, S.; Urn Models and their Application: An Approach to Modern Discrete Probability Theory. New York and London, Wiley, 1977. Wiley Series in Probability and Mathematical Statistics, 1977.

[11] dos Santos, J. P. O.; Mello, M. P. ; Murari, I.; Introdução à análise cominatória. Editora Ciência Moderna, primeira edição, 2008.

[12] Polya, G. Patterns of Plausible Inference. Princeton, N.J.: Princeton University Press, 1954.

[13] Ross, S.M.Probabilidade- Um Curso Moderno com Aplicações.Bookman, 2010.

[14] Sprott, D. A.; Probability distributions associated with distinct hits on targets, Bull. Math. Biophs., 19, 163-170, 1957.

[15] Stirzaker, D.; Elementary Probability.Cambridge University Press , 2003.

[16] Morgado, A.C.O.; Wagner, E.; Zani, S.; Progressões e Matemática Financeira. SBM, sexta edição, 2015. 
Valdivino Vargas Júnior Universidade Federal de Goiás <vvjunior@ufg.br>

Tiago Moreira Vargas Universidade Federal de Goiás <vargas@ufg.br>

Divaldo P. Fernandes Júnior Colégio Fractal $<$ profjr77@gmail.com>

Recebido: $14 / 02 / 2020$

Publicado: 21/08/2019 